\begin{tabular}{|c|c|}
\hline Citation & $\begin{array}{l}\text { Naveen Krishna Reddy, Christian Clasen, (2013), } \\
\text { Self-propelling micro-disks } \\
\text { Korea-australia Rheology Journal, 26(1), 73-79. }\end{array}$ \\
\hline Archived version & $\begin{array}{l}\text { Author manuscript: the content is identical to the content of the published } \\
\text { paper, but without the final typesetting by the publisher }\end{array}$ \\
\hline Published version & http://dx.doi.org/10.1007/s13367-014-0008-2 \\
\hline Journal homepage & Klik hier als u tekst wilt invoeren. http://link.springer.com/journal/13367 \\
\hline Author contact & your email christian.clasen@cit.kuleuven.be \\
\hline IR & url in Lirias https://lirias.kuleuven.be/handle/123456789/ 444450 \\
\hline
\end{tabular}

(article begins on next page) 


\title{
Self-propelling micro-disks
}

\author{
Naveen Krishna Reddy, Christian Clasen* \\ ${ }^{1}$ Department of Chemical Engineering, KU Leuven, W. de Croylaan 46, 3001 Leuven, \\ Belgium \\ *Corresponding author: christian.clasen@cit.kuleuven.be
}

\begin{abstract}
In this paper we introduce a simple and scalable method to produce micrometer sized 'Janus' disks whose rim is coated half with platinum/palladium and half with gold. The disks pinned upright to the air/liquid interface disks exhibit self-propulsion of $\sim 100 \mu \mathrm{m} / \mathrm{s}$ when submerged in $\mathrm{H}_{2} \mathrm{O}_{2}$ solution, due to the catalytic growth of oxygen bubbles at the disks upper (platinum/palladium-coated) rim. The disks exhibit two different travel trajectories, linear and rotary, depending on the bubble growth position, and are propelled via two different mechanisms, the bubble growth and the bubble burst. The displacement speed due to the bubble burst is three orders of magnitude larger than from the bubble growth process, whereas displacement distances are of similar order of magnitude for both processes.
\end{abstract}




\section{Introduction}

Nature uses self-propulsion to move objects from one place to another in a specific direction, for example towards sources of food. The size of natural self-propelling objects ranges from tens of meters (whales and elephants) to few microns (bacteria). There are, however, no nature made self-propelling particles in the nanometer range simply because the energy cost to overcome viscous drag at this scale is too large (Berg (1983), Berg (2004), Purcell (1977)). In contrast to this the size of man-made self-propelling particles has continuously been decreased over the last decade, starting from the first selfpropelled objects of centimeter and millimeter scale (Ismagilov et al., 2002), to few nanometer long rods (Paxton et al. (2004); Fournier-Bidoz et al. (2005)). Recently there has been a vast increase in the number of studies on the topic of self-propulsion, with particles of various shapes, sizes and propelling mechanisms. Large-scale objects on the millimeter and centimeter scale were studied by Whiteside's group (Ismagilov et al. 2002) who used a bubble recoil/detachment mechanism for self-propulsion driven by oxygen bubbles produced on a platinum catalyst in hydrogen peroxide solution. Hayashima et al. (2002) studied self-propelling millimeter sized camphanic acid disks at air/water interface. Recently Velev et al. used miniature semiconductor diodes to propel millimeter sized floating rafts using electric filed induce electro-osmotic flow (Chang et al. (2007)). Apart from the above few studies on large objects most of the work in literature currently focuses on nanoscale particles (Ebbens and Howse (2010)). The challenge to produce self-propelling nanomotors increases with decreasing length scale as they have to generate sufficient forces to overcome viscous drag (Berg (1983), Berg (2004), Purcell (1977)). In spite of these difficulties many researcher have produced nano- and micro-particles that can self- propel, examples are bimetallic nanorods (Paxton et al. (2004), Fournier-Bidoz et al. (2005), Reddy et al. (2013)), half platinum coated spheres (Howse et al. (2007)), rolled up microtubes (Solovev et al. (2009)), flexible artificial flagellum (Dreyfus et al. (2005)) or chiral colloidal propellers (Ghosh and Fischer (2009)). The above mentioned particles use a variety of self-propelling mechanisms such as bubble recoil/detachment (Ismagilov et al. (2002); Vicario et al. (2005), Stock et al. (2008), Gibbs and Zhao (2009)) bubble collapse (Manjare et al. (2012)), electro-osmosis (Chang et al. (2007)), self electrophoresis (Paxton et al. (2004), Fournier-Bidoz et al. (2005), Mano and Heller (2005), Wang et al. (2006)), selfdiffusiophoresis (Golestanian et al. (2005), Howse et al. (2007)) and flagella motion (Dreyfus et al. (2005)). However, most of the synthesis procedures are laborious and difficult to scale up in terms of number of particles produced.

So far only few methods have been introduced to produce self-propelling disks. All of these utilize solely disks pinned flat to the liquid/air interface (Ismagilov et al. (2002), Hayashima et al. (2002), Nakata et al. (2006), Nakata and Murakami (2010)). Whiteside's group used polydimethylsiloxane (PDMS) plates of 1-2 mm thickness and 9 $\mathrm{mm}$ diameter to which small a piece of platinum covered porous glass filter was attached at the bottom (Ismagilov et al. (2002)). The PDMS sheets were placed on a liquid/air interface such that the platinum covered glass filter was immersed in the liquid (1-3\% $\mathrm{H}_{2} \mathrm{O}_{2}$ solution). In another case camphanic acid disks were floated on a phosphate buffer solution where the propulsion was caused by a small dissolution of camphanic acid into the bulk (Hayashima et al. (2002)). 
In this paper we investigate for the first time the self-propulsion of micro-disks pinned upright at a liquid/air interface. The propulsion mechanism is based on catalytic conversion of $\mathrm{H}_{2} \mathrm{O}_{2}$ to water and oxygen by platinum/palladium $(\mathrm{Pt} / \mathrm{Pd})$ alloy and gold (Au) (Ismagilov et al. (2002), Stock et al. (2008), Gibbs and Zhao (2009)), and the upright disks are driven by oxygen bubble growth on the disks inside the liquid and their burst upon contact with the liquid/air interface. The synthesis of the disks is based on a simple method to produce 'Janus' rods that self-propel in the direction perpendicular to their long axis (Reddy et al. (2013)). Extending this technique we demonstrate in this paper the synthesis of 'Janus' micro-disks based on a precise microtome cutting. The design suggested here can be used to obtain self-propelling disks whose diameter can range from few micrometers to hundreds of micrometers and their thickness from few nanometers to few micrometers. The amount of self-propelling particles produced by this method is easily scalable to study the self-propulsion at larger particle concentrations.

\section{Materials and methods}

Micro-disks were fabricated by slicing aligned micro-rods using a microtome. The material used for the rod was human hair as an ubiquous and readily available material with reproducible dimensions. The diameter of a human hair ranges from $50-100 \mu \mathrm{m}$ and decreases linearly from the stem to the tip. For the current synthesis $1.5 \mathrm{~cm}$ from the central part of a $50 \mathrm{~cm}$ long hair has been selected to obtain a narrow diameter distribution of $\sim 100 \pm 10 \mu \mathrm{m}$. The procedure for preparing the micro-disks is shown in Figure 1. The rods were aligned and fixed onto a rigid polymer sheet as shown in Figure 1.a. The open spacing in the center of the polymer sheet gave access to both sides of the rods surface. The aligned rods were sputter coated on one side with platinum/palladium $(\mathrm{Pt} / \mathrm{Pd})$ alloy and on the other side with gold $(\mathrm{Au})$ using a sputter coater (Q150T, Quorum Technologies Ltd, UK), thus creating 'Janus' micro-rods with metal layers of 5 $\mathrm{nm}$ thickness as described in Reddy et al. (2013). The aligned bi-metal coated rods were subsequently placed in water and frozen before microtoming with a sliding microtome (Reichert, Austria). Via this approach Janus micro-disks with a thickness of $20 \mu \mathrm{m}$, coated on one half with $\mathrm{Pt} / \mathrm{Pd}$ and on the other half with $\mathrm{Au}$, were sliced out of the rods (Figure 1.b). It should be noted that this procedure locates the metallic coating not on the micro-disks faces but on the disks rim (Figure 1.a).

Self-propulsion experiments were carried out in a closed glass bottom culture dish (MatTeck corporation, USA). A small number of micro-disks were added to a $20 \mathrm{wt} . \%$ hydrogen peroxide $\left(\mathrm{H}_{2} \mathrm{O}_{2}\right)$ solution (CHEM-LAB, NV, Belgium) and their movement was observed in the surface plane with an inverted microscope (IX71, Oympus, USA) and perpendicular to the surface plane with a high-speed CMOS camera (FASTCAM SA2, Photron, San Diego, CA) equipped with a home-build long working distance microscopic objective. 
(a)

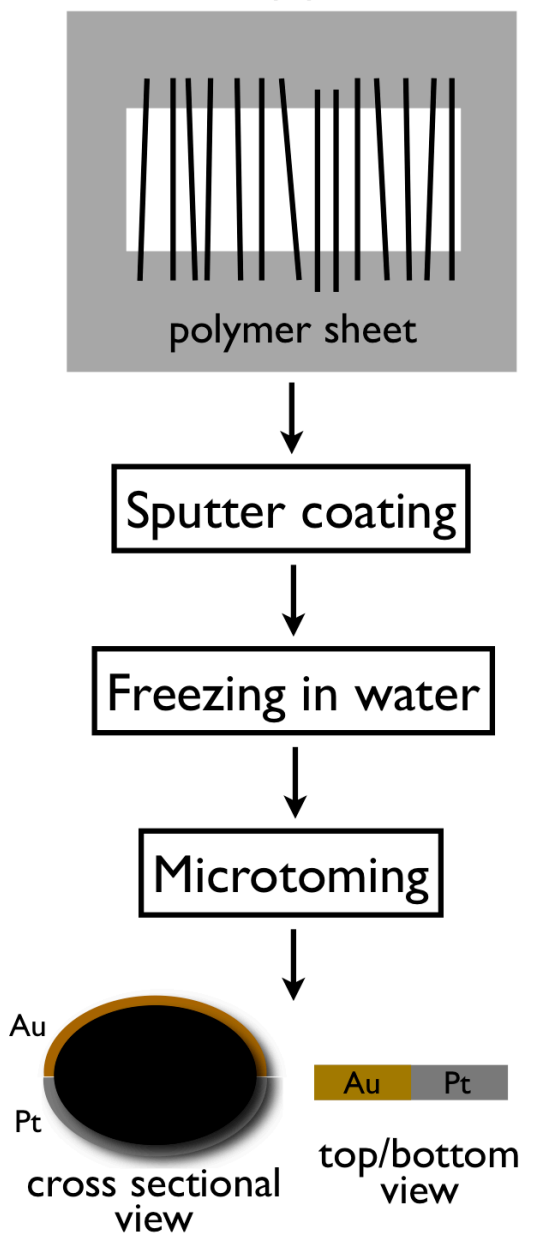

(b)
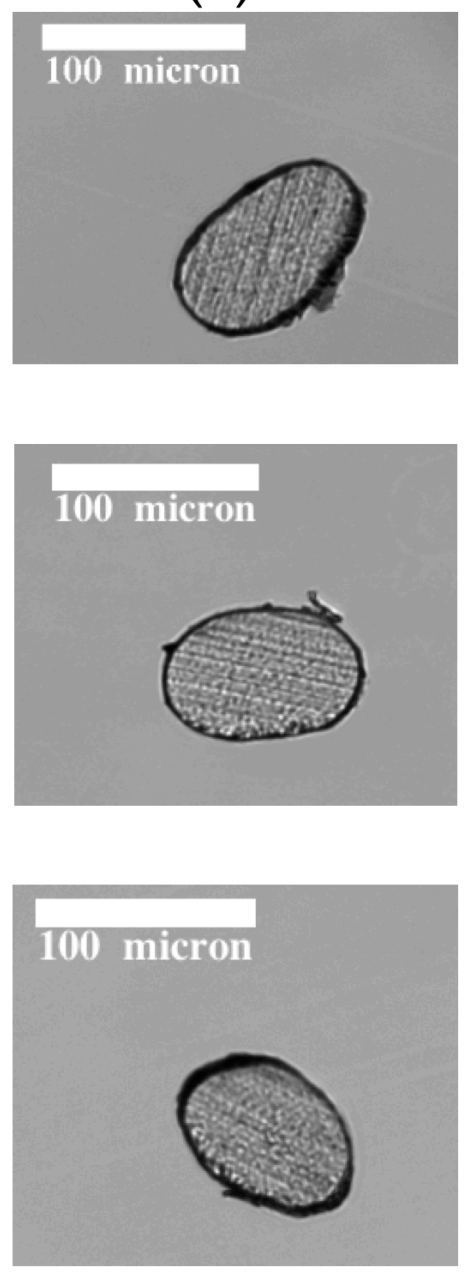

Figure 1: a) Schematic representation showing the 'Janus' disk preparation procedure. b) 'Janus' disks obtained after microtoming with approximately $100 \mu \mathrm{m}$ diameter and slightly elliptical shapes.

\section{Results}

The micro-disks when placed in water lie initially flat on the bottom of the container since their average density is greater than that of water (see Figure 1.b). In $\mathrm{H}_{2} \mathrm{O}_{2}$ solution the micro-disks surface coated with Pt catalyses the conversion of $2 \mathrm{H}_{2} \mathrm{O}_{2} \rightarrow$ $2 \mathrm{H}_{2} \mathrm{O}+\mathrm{O}_{2}$. At low reactant concentrations the dominant mechanism for propulsion from this reaction has been reported to result from self-diffusiophoresis (Reddy et al. (2013)) due to the combined catalytic effect of Pt and Au. 
However, in case that the rate of generation of oxygen becomes larger than the rate of diffusion into the solution, oxygen bubbles begin to grow on the micro-disks (see movies in the supplementary information). In this case the rate of oxygen generation is controlled by the available surface of the Pt catalyst. As the oxygen bubbles grow the micro-disks first tilt upright due to buoyancy such that their equatorial axis is perpendicular to the bottom glass plate and the liquid/air interface (see Figure 2).

(a)

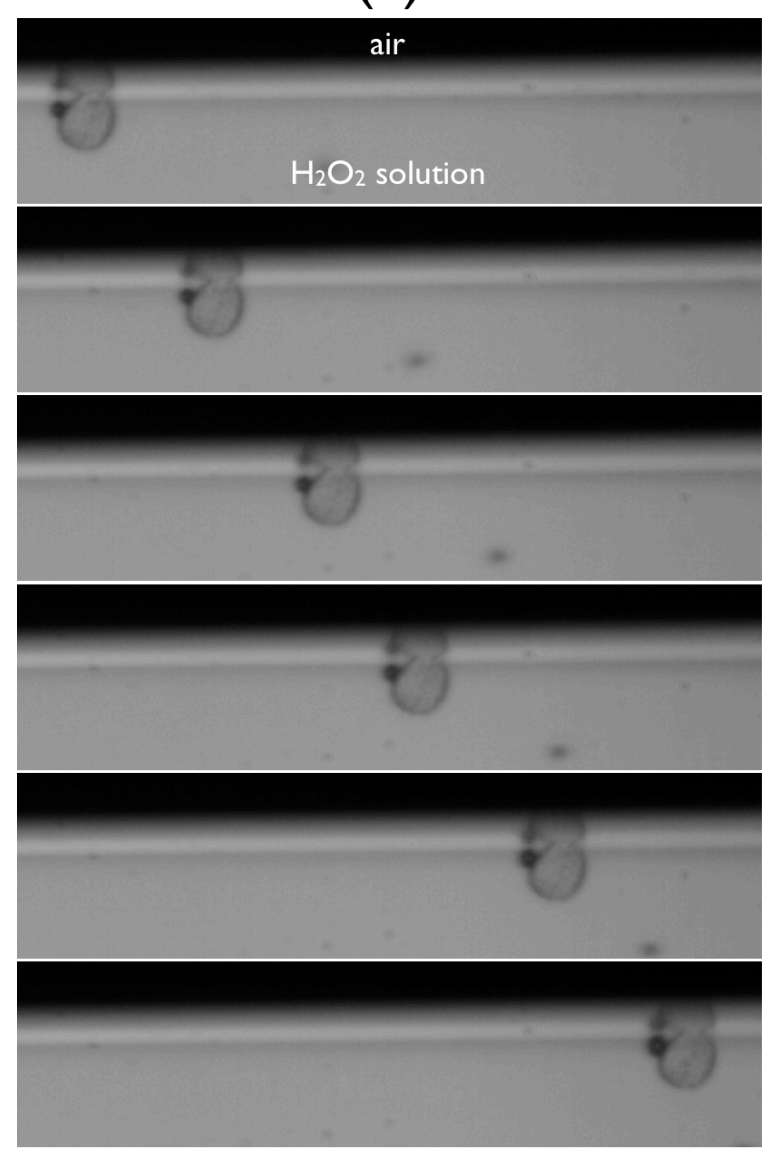

(b)

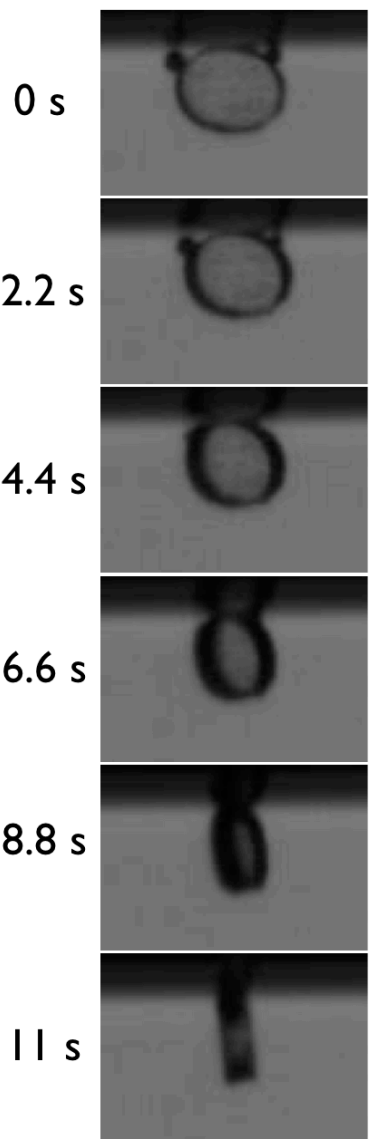

Figure 2: Sequence of images (side view) showing the self-propulsion of 'Janus' disks in $20 \mathrm{wt} \% \mathrm{H}_{2} \mathrm{O}_{2}$ solution at the liquid/air interface. The images are separated by time intervals of $2.2 \mathrm{~s}$ for clarity. The propulsion inducing a) linear and b) rotational motion is due to one and two oxygen bubbles created on the 'Janus' disks rim inside the $\mathrm{H}_{2} \mathrm{O}_{2}$ solution that are bursting when they come in contact with liquid/air interface. 
Subsequently the bubbles lift the upright micro-disk to the air/liquid interface. This process pins only a part of the $\mathrm{Pt} / \mathrm{Pd}$ covered disks rim to the air/liquid interface, leaving the rest of the upright disks surface inside the liquid phase. Once the disks rim is pinned to the interface two mechanisms are involved in its propulsion, oxygen bubble growth and burst. For the current study care was taken to avoid processes such as bacterial propulsion or convection that cause an apparent self-propelling. As the micro-disks produced here have a diameter of $100 \mu \mathrm{m}$ the above erroneous processes as well as Brownian motion can be neglected (Dunderdale et al. (2012)).

The first propulsion mechanism is based on a displacement of the disk due to bubble growth. During the bubble growth process, smaller oxygen bubbles are being generated on the portion of the rim inside the liquid that is coated with Pt. The smaller bubbles coalesce to form bigger bubbles that are still pinned to the disks rim and are fully immersed in the liquid phase (see Figure 2 and schematic in Figure 3).

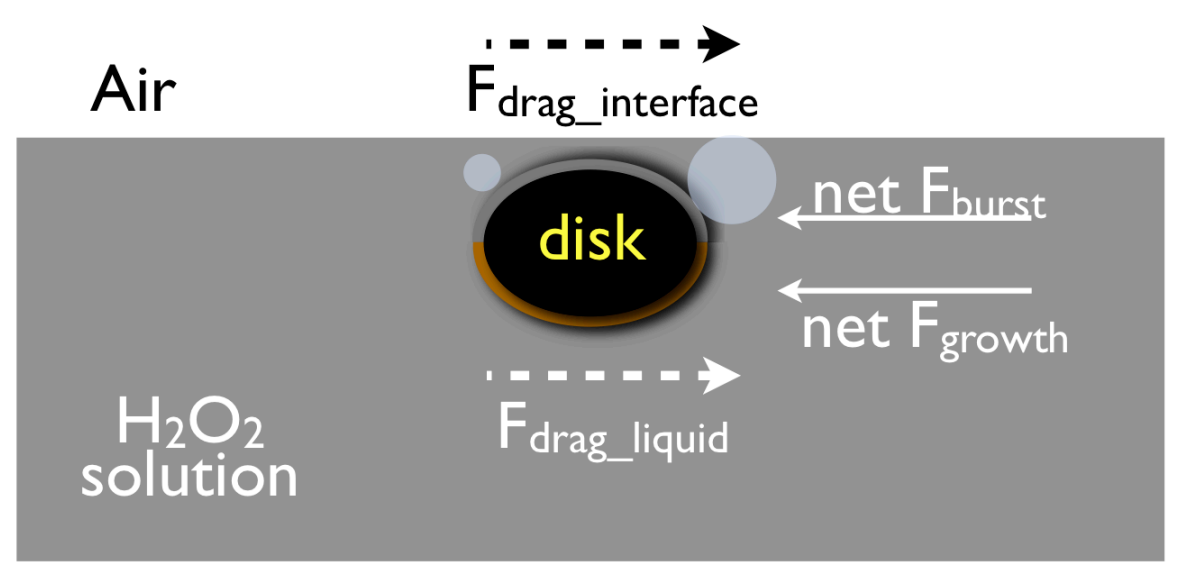

Figure 3: Schematic showing the forces acting on the micro-disk during self-propulsion at the air/liquid interface. The forward propulsion is due to asymmetric distribution of the oxygen bubble growth and burst. The drag on the self-propelling micro-disk is arising from the bulk liquid and the air/liquid interface.

As the oxygen bubble grows it pushes the disk away from the bubbles center thus propelling it to one side in a continuous motion. The second propulsion mechanism that follows the initial bubble growth is based on the burst of the bubble that occurs when the growing bubble eventually touches the air/liquid interface. In the oxygen bubble burst process the shock wave generated after the burst propels the disk away from the center of the bubble, causing ballistic propulsion. The bubble burst at the liquid/air interface contact has been studied extensively in literature (Bird et al. (2010), Liger et al. (2012)). The bubble burst mechanism is preceded by the bubble protruding out of the interface into the gas phase causing the liquid film on the hemispherical bubble to drain. The pressure inside the bubble is greater than the surrounding atmosphere due to bubbles curvature. Next a hole nucleates at the thinnest part of the film causing the liquid film to retract. The sudden depression forming at the interface bubbles former location generates a traveling shock wave at the interface. This traveling liquid wave propels the disk away 
from the original bubble location. The depression also causes other phenomenon such as the formation of daughter droplets and vertical jets depending on the Reynolds number, $\operatorname{Re}=\rho r U / \mu$ and the capillary number, $\mathrm{Ca}=\mathrm{U} \mu / \gamma$, where $\rho$ is the liquid density, $\mathrm{r}$ is the bubble radius, $\mathrm{U}$ is the speed of liquid film retracting after bubble burst, $\mu$ is the liquid viscosity and $\gamma$ is the surface tension between liquid and air (Bird et al. (2010)). The formation of daughter droplets that are ejected into the air is also visible in the movies (see supplementary information, M4-M5).

In principle there are two stable configurations possible for the bubbles pinned to the disk, which will result in two different propulsion trajectories. The first configuration is with the bubbles attached to the width of the rim, resulting in a linear trajectory (Figure 2.a). Although in principle each bubble burst results in a sudden acceleration, for most self-propelling disks there are several smaller bubbles growing at the rim that burst at different time intervals and cause an apparent continuous motion. The average velocity for micro-disks propelled linear by oxygen bubbles bursting at the liquid/air interface is $100 \mu \mathrm{m} / \mathrm{s}$ (Figure 2.a and movie M1). The forces acting on the linearly self-propelling micro-disks are shown schematically in Figure 3. The micro-disk in Figure 2.a is selfpropelled by an asymmetric distribution of the bubbles at the rim, resulting in a net force propelling the disk in one direction. The forces resisting this motion are caused by drag exerted on the micro-disk by the liquid and the contact to the liquid/air interface. The bulk of the micro-disk is inside the liquid and therefore this viscous drag is estimated to be larger than the drag from the liquid/air interface, which acts, only on the pinned part of the disk. The drag force on the disk moving at $100 \mu \mathrm{m} / \mathrm{s}$ can be calculated by neglecting the drag from the pinned part of the disk at the liquid/air interface. The viscous drag force on a disk moving perpendicular to the short axis of the disk is given by $\mathrm{F}_{\text {drag }}=$ $0.5 \rho \mathrm{C}_{\mathrm{DV}}{ }^{2} \mathrm{~A}$, where $\rho$ the density of the liquid, $\mathrm{C}_{\mathrm{D}}$ the drag coefficient, $\mathrm{v}$ and $\mathrm{A}$ are the velocity and total area of the disk. The drag coefficient of a disk facing the flow direction with its rim can be calculated for low Reynolds numbers from $C_{D}=64(1+\operatorname{Re} / 2 \pi) / \pi R e=$ 3045 (Clift et al. (1978)), where $\mathrm{Re}=\mathrm{d}_{\mathrm{e}} \mathrm{U} \rho / \mu$, with $\mathrm{d}_{\mathrm{e}}=\left(6 \mathrm{a}^{2} \mathrm{H}\right)^{1 / 3}$ the effective diameter of an equivalent sphere, where $\mathrm{a}$ is the radius and $\mathrm{H}$ the thickness of the disk. For the present case with an effective disk area of $A=1.4 \times 10^{-8} \mathrm{~m}^{2}$ we have $\mathrm{Re}=0.0067$. The drag force on the micro-disk that is overcome by the bubble propulsion is thus $21 \times 10^{-9}$ $\mathrm{N}$.

The second stable configuration for growing bubbles leads to a rotary trajectory of the disk. In this case the bubble is still in contact to the edge of the catalysing disks rim, but mainly pinned to the side of the disk (Figure 2.b). The side pinning is promoted by a) a light deviation of the fibers from the cylindrical shape and b) a slight edge deformation resulting from the microtoming such that they protrude out (Figure 1.b and Figure 4). Similar to the linear travel the net force leading to the rotation of the disks seen in Figure 4 is caused by an asymmetric distribution of the growth and burst of bubbles as shown in Figure 4.a for the case of two bubbles and Figure 4.b for a single bubble. A coupling of the two stable configurations of growing bubbles from the edge of the disk's rim as well as from the disk's side leads to rotation coupled with a small amount of linear motion. 


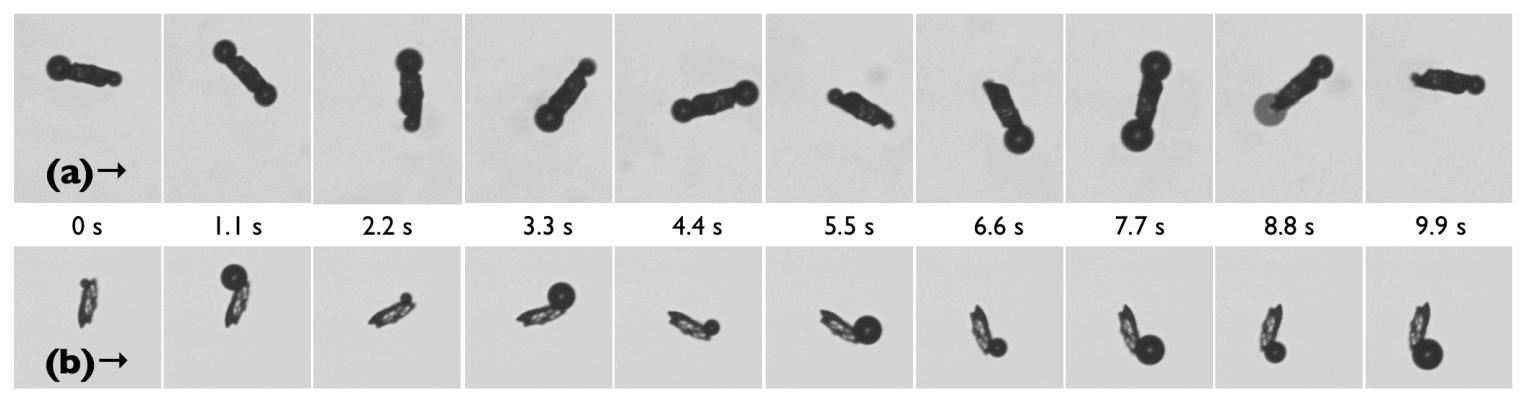

Figure 4: Sequence of images (bottom view), showing the rotational motion of 'Janus' disks in $20 \mathrm{wt} \% \mathrm{H}_{2} \mathrm{O}_{2}$ solution. The images are separated by a time interval of $1.1 \mathrm{~s}$ for clarity. a) Propulsion of 'Janus' disk by oxygen bubbles growing on opposite sides of the disk, b) a single oxygen bubble growing only on one side of the disks edge. Movies of the rotating disks shown here (taken at a frame rate of 9 frames/s) are provided in the supplementary information (M4-M5).

The observed average angular velocity of the micro-disk propelled by two opposing bubbles is $\sim 2 \pi \mathrm{rad} / \mathrm{s}$. The data obtained by tracking this rotational motion of the disks is plotted in Figure 5. Again similar to the linear travel the combined effects of several bubble bursting at infrequent intervals imparts a smooth rotational torque on the disk, and leads to an apparently continuous motion as seen in Figure 5.a. A quantitative analysis and separation of the two propulsion mechanism is possible for the case of a single growing bubble as depicted in Figure 4.b and a clear separation of an initially smooth and continuous motion during the bubble growth process and a sudden ballistic motion immediately after the burst is observed. The resulting angular displacement profile can be seen in Figure 5.b. 

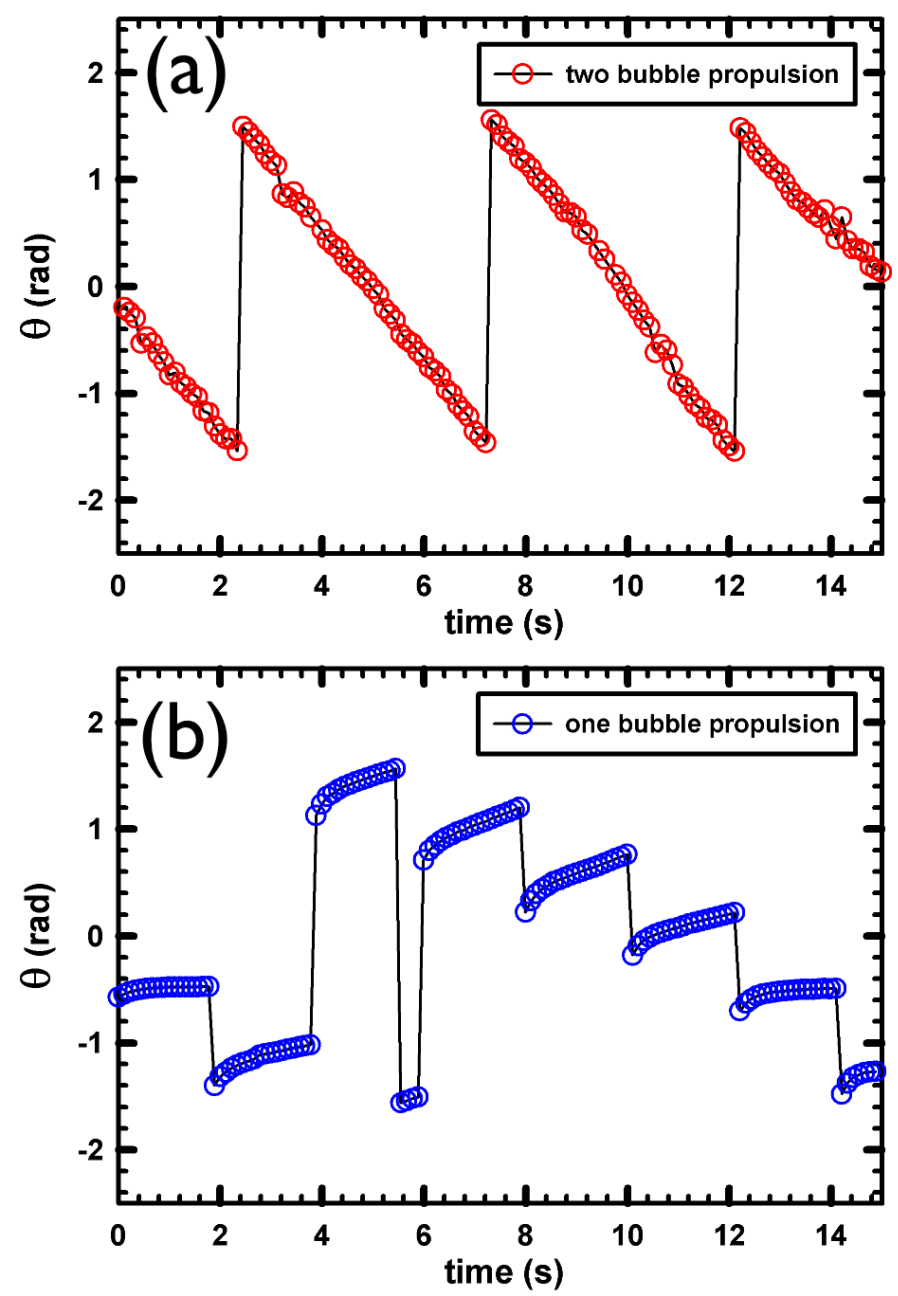

Figure 5: Angular displacement of 'Janus' disks in radians as a function of time due to a) propulsion by two bubbles at the opposite ends of the disk and b) propulsion by one bubble. We can clearly see the smooth rotation in the case of propulsion by two opposing bubbles and an abrupt motion in the case of propulsion by one bubble.

For the case of a single growing bubble the initially several small bubbles quickly coalesce into a single one that always growths from the same pinning point on the disk's rim. The disk is pushed to the side by the expanding bubble that occupies the space previously taken by the disk. This causes the disk to self-propel at the speed of the oxygen bubble growth as shown in Figure 6.a. The angular displacement of the disk in Figure 5.b due to the bubble growth is approximately equal to the radius of the growing bubble over the distance of the pinning point to the rotational axis. 


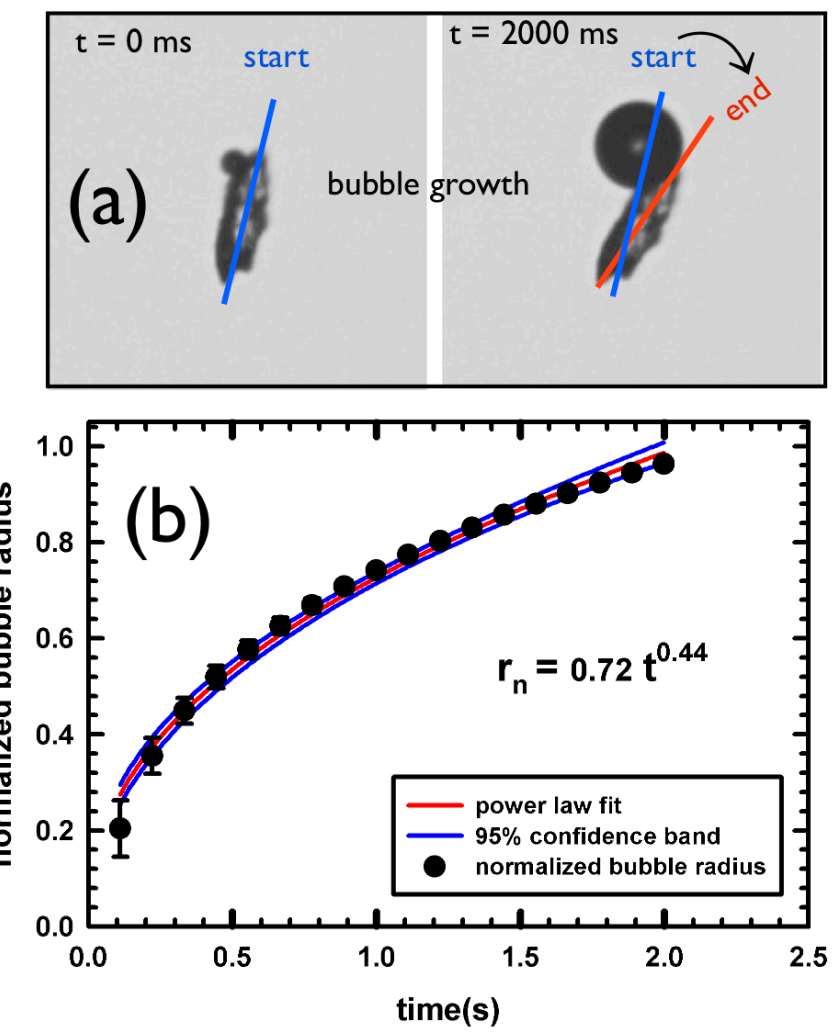

Figure 6: a) Snapshots of bubble growth at time $\mathrm{t}=0 \mathrm{~ms}$ and just before bubble burst $(\mathrm{t}=$ $2000 \mathrm{~ms}$ ). b) Oxygen bubble growth process plotted as normalized bubble radius as a function of time. The filled symbols are experimental data and the line is a power law fit. Also shown is the $95 \%$ confidence band for the power law fit.

The bubble growth process follows a power law scaling, with $\mathrm{R}_{\text {growth }}=\mathrm{at}^{\mathrm{n}}$ (Figure 6.b). The power law exponent for the bubble growth data obtained from Figure 6.b is $n=0.44$. The exponent for the bubble growth depends on the growth process and varies between 0.33 to 0.50 (Thorncroft et al. (1998)). An exponent of $n=0.33$ is observed for an energy depletion mechanism, whereas $\mathrm{n}=0.5$ is seen for a diffusion controlled growth. The observed value from the fit of the data in Figure 6.b of $n=0.44$ indicates a mixed bubble growth mechanism with a dominant diffusion control. The average propulsion velocity over a single bubble growth cycle of $3 \mu \mathrm{m} / \mathrm{s}$ (resulting for the current case in an average angular velocity of $0.25 \mathrm{rad} / \mathrm{s}$ ) is slow in comparison to the subsequent burst propulsion. Figure 7 shows a sequence of two images of a micro-disk before and after bubble burst. 


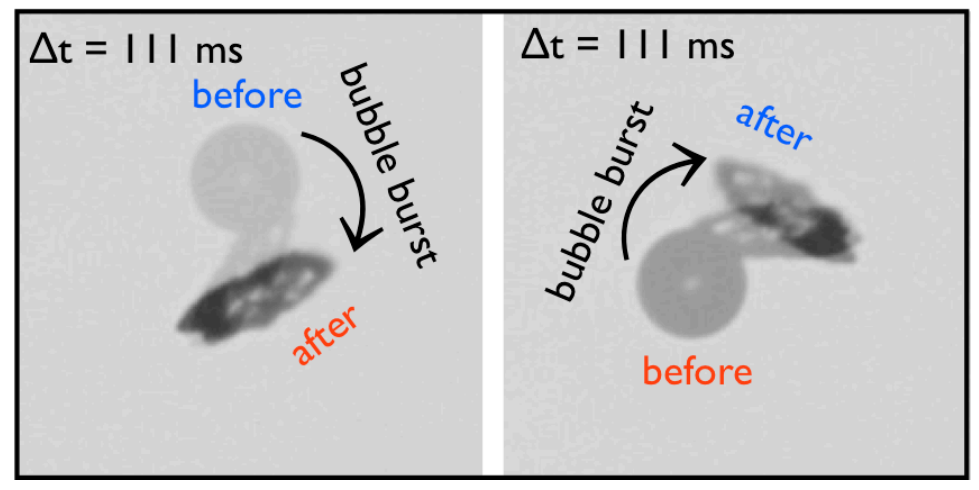

Figure 7: Two consecutive images of the bubble burst process, showing the bubble just before the bubble burst and immediately after the burst. The time interval between the images is $111 \mathrm{~ms}$.

The direction of the displacement due to the bubble burst is the same as that for the bubble growth process. The time interval between the two frames is $111 \mathrm{~ms}$, indicating that the bubble burst process takes less than this time scale. Recently the time for the burst process of a gas bubble upon gas/liquid interface contact was detected to be around $10 \mathrm{~ms}$ using a high-speed camera (Manjare et al. (2012)). The velocity of the disk due to the oxygen bubble burst of $\sim 350 \mu \mathrm{m} / \mathrm{s}$ (Figure 8.b) is much faster than that originating from the bubble growth. However, the displacement $(\sim 80 \mu \mathrm{m}$, Figure $8 . \mathrm{a})$ is of similar order of magnitude compared to the bubble growth process.

\section{Conclusions}

We have shown a simple and scalable method to produce 'Janus' micro-disks whose rims are coated with platinum/palladium and gold. Ehen placed in $\mathrm{H}_{2} \mathrm{O}_{2}$ solution these disks of $\sim 100 \mu \mathrm{m}$ diameter and $10 \mu \mathrm{m}$ thickness are lifted to the air/liquid interface by buoyancy due to the catalytic growth of oxygen bubbles at the disks upper ( $\mathrm{Pt} / \mathrm{Pd}$-coated) rim. The disks are pinned upright with their rim to the liquid/air interface. The disks are propelled via two different mechanisms, the bubble growth and the burst. During oxygen bubble growth, the disks are propelled smoothly and the displacement is approximately equal to the radius of the oxygen bubble size. After the oxygen bubble bursts the disks are propelled ballistically. The displacement speed due to the bubble burst is three orders of magnitude larger than from the bubble growth process, whereas average displacement distances per time are of similar order of magnitude for both processes. 

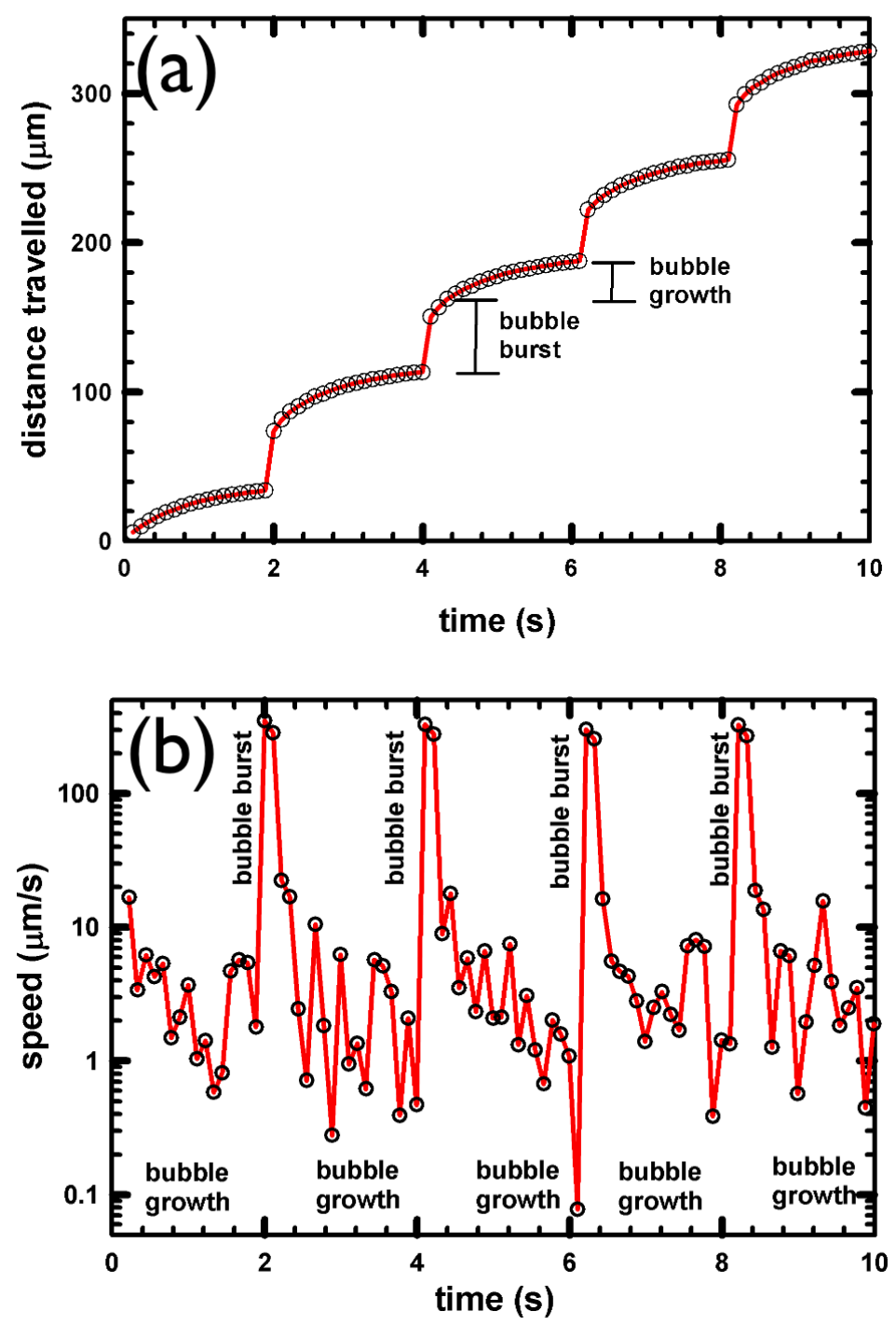

Figure 8: a) Distance travelled by the 'Janus' disks due to oxygen bubble growth and burst processes, for the propulsion due to bubble growth on the side of the disk. b) Same data as in (a) showing the propulsion speed.

\section{Acknowledgements}

The authors would like acknowledge financial support from the SoPPom program of the Flemish Strategic Initiative for Materials (SIM), as well as from the FWO (Research Foundation Flanders, FWO project G.0543.10N), and would like to thank Sylvie Van Loon, Ljiljana Palangetic, Wouter Mathues, Kasper Van Acker and Jan Fransaer for fruitful discussions. 


\section{References}

[1] Berg, H.C., 1983, Random Walks in Biology, Princeton University Press.

[2] Berg, H.C., 2004, E. coli in Motion, (Biological and Medical Physics, Biomedical Engineering), Springer-Verlag, New York Inc.

[3] Bird, J.C., Ruiter, R-de., Courbin, L. and Stone, H.A., 2010, Daughter bubble cascades produced by folding of ruptured thin films, Nature $465,759-762$

[4] Chang, S.T., Paunov, V.N., Petsev, D.N. and Velev, O.D., 2007, Remotely powered self-propelling particles and micropumps based on miniature diodes, Nature Materials $\mathbf{6}$, 235-240.

[5] Clift, R., Grace, J.R. and Weber, M.E., 1978, Bubbles, Drops, and Particles, Academic Press Inc, London.

[6] Dreyfus, R., Baudry, J., Roper, M.L., Fermigier, M., Stone, H.A., and Bibette, J., 2005, Microscopic artificial swimmers, Nature 437, 862-865.

[7] Dunderdale, G., Ebbens, S., Fairclough, P. and Howse, J.R., 2012, Importance of Particle Tracking and Calculating the Mean-Squared Displacement in Distinguishing Nanopropulsion from Other Processes, Langmuir 28, 10997-11006.

[8] Ebbens, S.J. and Howse, J.R., 2010, In pursuit of propulsion at the nanoscale, Soft Matter 6, 726-738.

[9] Fournier-Bidoz, S., Arsenault, A.C., Manners, I., and Ozin, G.A., 2005, Synthetic self-propelled nanorotors, Chem. Commun. 441-443.

[10] Ghosh, A. and Fischer, P., 2009, Controlled Propulsion of Artificial Magnetic Nanostructured Propellers, Nano Lett. 9, 2243-2245.

[11] Gibbs, J.G. and Zhao, Y.-P., 2009, Autonomously motile catalytic nanomotors by bubble propulsion, Appl. Phys. Lett. 94, 163104-1-3.I

[12] Golestanian, R. Liverpool, T.B. and Ajdari, A., 2005, Propulsion of a Molecular Machine by Asymmetric Distribution of Reaction Products, Phys. Rev. Lett. 94, 220801$1-4$.

[13] Hayashima, Y., Nagayama, M., Doi, Y., Nakata, S., Kimurac, M. and Iidac, M., 2002, Self-motion of a camphoric acid boat sensitive to the chemical environment, Phys. Chem. Chem. Phys. 4, 1386-1392. 
[14] Howse, J.R., Jones, R.A.L., Ryan, A.J., Gough, T., Vafabakhsh, R., and Golestanian, R., 2007, Self-Motile Colloidal Particles: From Directed Propulsion to Random Walk, Phys. Rev. Lett. 99, 048102-1-4.

[15] Ismagilov, R.F., Schwartz, A., Bowden, N. and Whitesides, G.M., 2002, Autonomous Movement and Self-Assembly, Angew. Chem. Int. Ed. 41, 652-654.

[16] Liger-Belair, G. Seon, T. and Antkowiak, A., 2012, Collection of collapsing bubble driven phenomena found in champagne glasses, Bubble Sci. Eng. Technol. 4, 21-34.

[17] Manjare, M., Yang, B. and Zhao, Y.-P., 2012, Bubble Driven Quasioscillatory Translational Motion of Catalytic Micromotors, Phys. Rev. Lett. 109, 128305-1-5.

[18] Mano, N. and Heller, A., 2005, Bioelectrochemical Propulsion, J. Am. Chem. Soc. 127, 11574-11575.

[19] Nakata, S., Kirisaka, J., Arima, Y. and Ishii, T., 2006, Self-Motion of a Camphanic Acid Disk on Water with Different Types of Surfactants, J. Phys. Chem. B. 110, 2113121134.

[20] Nakata, S. and Murakami, M., 2010, Self-Motion of a Camphor Disk on an Aqueous Phase Depending on the Alkyl Chain Length of Sulfate Surfactants, Langmuir 26, 24142417.

[21] Paxton, W.F., Kistler, K.C., Olmeda, C.C., Sen, A., St. Angelo, S.K., Cao, Y., Mallouk, T. E., Lammert, P.E., and Crespi, V.H., 2004, Catalytic Nanomotors: Autonomous Movement of Striped Nanorods, J. Am. Chem. Soc. 126, 13424-13431.

[22] Purcell, E.M., 1977, Life at Low Reynolds Number, Am. J. Phys. 45, 3-11.

[23] Reddy, N.K., Palangetic, L., Stappers, L., Buitenhuis, J., Fransaer J. and Clasen, C., 2013, Metallic and bi-metallic Janus nanobers: electrical and self-propulsion properties, J. Mater. Chem. C 1, 3646-3650.

[24] Solovev, A.A., Mei, Y. F., Urena, E.B., Huang, G.S., Schmidt, O.G., 2009, Catalytic Microtubular Jet Engines Self-Propelled by Accumulated Gas Bubbles. Small 5, 16881692.

[25] Stock, C., Heureux, N., Browne, W.R. and Feringa, B.L., 2008, Autonomous Movement of Silica and Glass Micro-Objects Based on a Catalytic Molecular Propulsion System, Chem. Eur. J. 14, 3146-3153.

[26] Thorncrofta, G.E., Klausnera, J.F and Mei, M., 1998, An experimental investigation of bubble growth and detachment in vertical upflow and downflow boiling. Int. Int. J. Heat Mass Transfer 41, 3857- 3871. 
[27] Vicario, J., Eelkema, R., Browne, W.R., Meetsma, A., La Crois, R.M. and Feringa, B.L., 2005, Catalytic molecular motors: fuelling autonomous movement by a surface bound synthetic manganese catalase. Chem. Commun. 3936-3938.

[28] Wang, Y., Hernandez, R.M., Bartlett, D.J., Bingham, Jr. J.M., Kline, T.R., Sen, A. and Mallouk, T.E., 2006, Bipolar Electrochemical Mechanism for the Propulsion of Catalytic Nanomotors in Hydrogen Peroxide Solutions, Langmuir 22, 10451-10456. 